\title{
Stage-by-Stage and Parallel Flow Path Compressor Modeling for a Variable Cycle Engine
}

\author{
George Kopasakis, ${ }^{1}$ Joseph W. Connolly ${ }^{2}$ \\ NASA Glenn Research Center, Cleveland, Ohio, 44135 \\ Larry Cheng $^{3}$ \\ University of Washington, Seattle, Washington, 98105
}

This paper covers the development of stage-by-stage and parallel flow path compressor modeling approaches for a Variable Cycle Engine. The stage-by-stage compressor modeling approach is an extension of a technique for lumped volume dynamics and performance characteristic modeling. It was developed to improve the accuracy of axial compressor dynamics over lumped volume dynamics modeling. The stage-by-stage compressor model presented here is formulated into a parallel flow path model that includes both axial and rotational dynamics. This is done to enable the study of compressor and propulsion system dynamic performance under flow distortion conditions. The approaches utilized here are generic and should be applicable for the modeling of any axial flow compressor design.

$\begin{array}{rlll} & & & \text { Nomenclature } \\ A & = & & \text { area }\left(\mathrm{m}^{2}\right) \\ c_{p} & = & & \text { specific heat at constant pressure }(\mathrm{J} / \mathrm{kg} \mathrm{K}) \\ F & = & & \text { flux term } \\ g & = & \text { gravitational constant, } 1\left(\mathrm{~kg} \mathrm{~m} / \mathrm{N} \mathrm{s}^{2}\right) \\ J & = & \text { total number of stages } \\ l & = & \text { length }(\mathrm{m}) \\ M & = & \text { Mach number } \\ N & = & \text { shaft rotational speed }(\mathrm{RPM}) \\ N_{c} & = & \text { corrected speed ratio } \\ n & = & \text { compressor stage number } \\ P & = & \text { pressure }\left(\mathrm{N} / \mathrm{m}^{2}\right) \\ r & = & \text { compressor radius }(\mathrm{m}) \\ R & = & \text { universal gas constant }(287 \mathrm{~N} \mathrm{~m} / \mathrm{kg} \mathrm{K}) \\ S & = & \text { source term } \\ T & = & \text { temperature }(\mathrm{K}) \\ T_{r} & = & \text { temperature ratio } \\ u & = & \text { axial flow velocity }(\mathrm{m} / \mathrm{s}) \\ V & = & \text { volume }\left(\mathrm{m}^{3}\right) \\ W & = & \text { rotational flow velocity }(\mathrm{rad} / \mathrm{s}) \\ \dot{W} & = & \text { mass flowrate }(\mathrm{kg} / \mathrm{s}) \\ W & = & \text { state term } \\ \dot{W}_{c m} & = & \text { mass flowrate }(\mathrm{kg} / \mathrm{s}) \\ & & \end{array}$

Greek

$\begin{array}{lll}\beta & = & \text { area weighted factor } \\ \gamma & = & \text { specific heat ratio }\end{array}$

\footnotetext{
${ }^{1}$ Senior Research Engineer, Intelligent Control and Autonomy Branch, 21000 Brookpark Rd.

${ }^{2}$ Aerospace Research Engineer, Intelligent Control and Autonomy Branch, 21000 Brookpark Rd.

${ }^{3}$ Graduate Student, University of Washington.
} 


$\begin{array}{lll}\eta & = & \text { efficiency } \\ \rho & = & \text { density }\left(\mathrm{kg} / \mathrm{m}^{3}\right) \\ \varphi & = & \text { variable associated with rotational dimension }\end{array}$

\begin{tabular}{|c|c|c|}
\hline \multicolumn{3}{|c|}{ Subscripts } \\
\hline$b$ & $=$ & variable associated with compressor bleed rate \\
\hline$C$ & $=$ & variable associated with stage characteristic \\
\hline$c b$ & $=$ & variable associated with combustor \\
\hline$c p$ & $=$ & variable associated with compressor \\
\hline$d$ & $=$ & variable associated with design value \\
\hline$j$ & $=$ & variable associated with state index $(j=1 . .4)$ \\
\hline$k$ & $=$ & number of compressor stages \\
\hline $\operatorname{lmp}$ & $=$ & variable associated with lumped volume \\
\hline$m$ & $=$ & parallel flow path number $(m=1 . . q)$ \\
\hline$m v$ & $=$ & variable associated with mixing volume \\
\hline$n$ & $=$ & compressor stage number $(n=1 \ldots 7)$ \\
\hline ref & $=$ & reference value of variable \\
\hline$S$ & $=$ & variable associated with static conditions \\
\hline$s t$ & $=$ & variable associated with compressor stages \\
\hline$s v$ & $=$ & static condition associated with stage volume \\
\hline$t$ & $=$ & variable associated with total conditions \\
\hline$t c$ & $=$ & total condition associated with stage characteristic \\
\hline$t v$ & $=$ & total condition associated with stage volume \\
\hline$x$ & $=$ & variable associated with the axial dimension \\
\hline
\end{tabular}

\section{Introduction}

upersonic vehicles are typically slender body aircraft with potentially pronounced structural flexibility modes. These modes can become exacerbated with the coupling of the propulsion system dynamics via thrust variations to cause performance challenges for vehicle ride quality and stability. ${ }^{1,2}$ The NASA Commercial Supersonic Technology (CST) Project under the Advanced Air Vehicles Program seeks to develop the technologies for commercial overland supersonic flight. Among several technical challenges is a task devoted to study AeroPropulsoServoElasticity (APSE), which deals with the study of vehicle dynamic performance. The propulsion system model utilized so far for APSE studies is based on 1-dimensional (1D) lumped volume component models with either lumped or quasi-1D models for the inlet and nozzle. ${ }^{2}$ The question remains, however, if lumped volume dynamic models provide sufficient fidelity for APSE dynamics, or if instead stage-by-stage or parallel flow path modeling may be necessary. Thus, the purpose of the stage-by-stage compressor modeling is to provide for more dynamic accuracy for investigating propulsion and APSE dynamic performance. The purpose of parallel flow path modeling is to provide the framework for more accurate engine component modeling to address the effects of flow distortion on the propulsion and APSE performance.

In the past, a form of volume dynamics stage-by-stage compressor model has been developed and validated utilizing specific compressor test data. ${ }^{3,4}$ This model, called Dynamic Turbine Engine Compressor Code (DYNTECC), ${ }^{5}$ has parallel compressor modeling capability that allows for radial and circumferential passage of mass and energy in a steady state fashion. Modeling in DYNTECC is based on an experimental compressor, and no techniques were developed to derive generic stage-by-stage models starting from a lumped volume approach, which would be desirable for propulsion system modeling prior to availability of hardware. Moreover, the parallel compressor model capability in DYNTECC does not include the rotational dynamics (except for a time lag). Besides, this model is deemed too complex to use for an overall engine simulation. Earlier, the Greitzer model ${ }^{6}$ was developed for predicting stall and surge behavior. These previous models did not meet the current needs, primarily due to not including the rotational dynamics that may become important relative to the overall APSE objectives in addressing the effects of flow distortion.

This paper describes the methodology of developing stage-by-stage and parallel flow path models starting from a generic lumped volume compressor model. The methodology outlines the step-by-step process for developing sub-compressor models 
for a stage-by-stage compressor model, and then extending this model to also develop circumferential sub-compressor models for each stage in order to develop a parallel flow path compressor model. The stage-by-stage and parallel flow path models are compared for validation and accuracy against the lumped volume compressor model. The technique for lumped volume compressor modeling has been already validated in both steady state and dynamically against compressor engine test data. ${ }^{7}$ The stage-by-stage compressor model developed here is qualitatively compared to another stage-by-stage model found in literature. ${ }^{5}$ The compressor model in this effort has been extended to cover stall and surge behavior. The objective in this effort is to capture the compressor dynamics while the compressor operation approaches surge and while it enters into stall conditions.

The compressor models that are developed here are for the High Pressure Compressor (HPC) of a Variable Cycle Engine (VCE) concept for supersonic vehicle research. This VCE engine has 3 compression systems and the HPC for which these models are developed has 7-stages. However, the approaches developed for stage-by-stage and parallel flow path compressor modeling are generic, and can be applied to any axial compressor design. The results presented here are for the isolated 7-stage HPC component models for this VCE concept.

The paper is organized as follows. First, the compressor modeling approach for stage-by-stage, followed by parallel flow path modeling are covered. This is followed by simulation results that compare the performance of the stage-by-stage and the parallel flow path compressor models to the respective lumped volume model, and comparison responses for time domain and frequency domain are shown. Finally, some concluding remarks are provided.

\section{Stage-by-Stage Compressor Model}

In the stage-by-stage compressor modeling approach, the lumped volume compressor maps are scaled down in a series of steps to develop a single stage model of appropriate size. The single stage models are then successively stacked and the cross sectional area of each of the stages is computed to develop an overall stage-by-stage compressor model. Through this process, this stacked model is stable and achieves the same steady state performance as the original lumped volume compressor model.

\section{A. Stage Volume Dynamics}

System dynamics are modeled for the stage volumes by the conservation of mass, momentum, and energy. Assuming perfect gas, adiabatic and compressible flow, the Euler formulations remain the same as those utilized for the lumped volume compressor model. ${ }^{7}$ The equations for mass, axial momentum, energy, and the state equation, respectively, are as follows.

$$
\begin{gathered}
\frac{d}{d t} \rho_{s v, n}=\frac{1}{V_{n}}\left(\dot{W}_{c, n}-\dot{W}_{c, n+1}-\dot{W}_{b, n}\right) \\
\frac{d}{d t} \dot{W}_{c, n}=\frac{A_{n} g}{l_{n}}\left(P_{t c, n}-P_{t v, n}\right)\left(1+\frac{\gamma_{c p}-1}{2} M_{m v}^{2}\right)^{\frac{-\gamma_{c p}}{\gamma_{c p}-1}} \\
\frac{d}{d t}\left(\rho_{s v, n} T_{t v, n}\right)=\frac{\gamma_{c p}}{V_{n}}\left(T_{t c, n} \dot{W}_{c, n}-T_{t v, n} \dot{W}_{c, n+1}-T_{t v, n} \dot{W}_{b, n}\right) \\
P_{t v, n}=\left(1+\frac{\gamma_{c p}-1}{2} M_{n}^{2}\right)^{\frac{1}{\gamma_{c p}-1}} \rho_{s v, n} R T_{t v, n}
\end{gathered}
$$

For other supporting equations that complete the mathematical description of the stage element models see Ref. [7].

The lumped volume performance characteristics are scaled appropriately to derive the stage performance characteristics for the stage-by-stage compressor model, which will be covered in the next subsection. If the stage performance of the compressor were known, these performance data would be used directly to construct the stage models and this scaling step would be skipped.

\section{B. Initial Scaling}

The stage performance characteristics (maps) will dictate the steady state conditions of the compressor. Equivalent stage compressor maps for simulation can be derived by scaling the lumped volume compressor maps, which may be available either from cycle analysis using the Numerical Propulsion System Simulation (NPSS), ${ }^{8}$ generically derived from a respective compressor map generation routine, or may be available from overall compressor testing. It is important to properly scale these maps so that the correct outlet conditions are met for each compressor stage, and the overall steady state performance of the stage-by-stage compressor matches that of the respective lumped volume model. In this case, lumped compressor performance characteristics, initially available through an NPSS model of the engine and utilized to develop a lumped compressor dynamic model, were scaled as follows. 


$$
\begin{aligned}
P_{r, s t} & =\sqrt[J]{P_{r, l m p}} \\
\eta_{s t} & =\sqrt[J]{\eta_{l m p}} \\
l_{s t} & =\frac{1}{J} l_{l m p} \\
\dot{W}_{s t} & =\dot{W}_{l m p} \\
\dot{W}_{b, s t} & =\frac{1}{J} \dot{W}_{b, l m p}
\end{aligned}
$$

Here, $J$ is the total number of stages. As will be described later in detail, the scaling for pressure ratio, efficiency, and corrected mass flow were all fine-tuned at a later point to improve the steady state accuracy of the model. There are four bleed flows extracted from this VCE HPC, which are scheduled based on operating conditions. For simplicity, it is assumed in this model that the bleed rates are equally distributed among compressor stages (Eq. (9)). The equal distribution of bleeds among compressor stages is not anticipated to have a significant impact on the results and it can be easily changed to match a specific configuration. Based on the equal distribution of bleeds simplification, the mass flowrate for each compressor stage coming from the downstream direction can be calculated as follows.

$$
\dot{W}_{c, n}=\dot{W}_{l m p}-n * \dot{W}_{b, s t}
$$

The Mach number can be approximated for each stage as

$$
M_{n}=M_{\text {in }}-\frac{n}{J}\left(M_{\text {in }}-M_{\text {out }}\right)
$$

Here, $M_{\text {in }}$ and $M_{\text {out }}$ refer to the Mach number in and out of the lumped volume model, respectively.

The first step is to develop a scaled down, single stage element of the lumped volume model. Doing so in one step, however, will result in an unstable model. This is because the initial conditions required for asymptotic convergence to an operating point for the single element model will be too different from those utilized to run the lumped volume model. Therefore, starting with the lumped volume compressor model, an intermediate scaling process is used as necessary to converge on stable initial conditions by scaling down, for example, by $3 / 4,1 / 2,1 / 3,1 / 4, \ldots, 1 / 7$. By running the scaled model at each step, final state conditions are obtained, which are then substituted for initial conditions in order to minimize the startup transients. It may take two or three runs/substitutions for the same scaled model to converge to initial conditions with a sufficiently small startup transient, which allows for larger subdividing steps for the next scale down step of the single stage element model.

Each of the VCE compression systems (3 in this case) comes with its own Variable Guide Vane (VGVs), whose angles are set based on an operating schedule that is derived in this case by using NPSS cycle analysis. The compression system performance characteristics (maps) change based on the VGV angle setting. To simulate this performance, typically, two sets of maps are utilized (derived using NPSS), which simulate the performance at the two extreme VGV angle settings, such as $0^{\circ}$ and $45^{\circ}$. In order to simulate different VGV angles, these maps are interpolated accordingly. The same set of maps used for the lumped compressor model are also utilized for the stage-by-stage model, and these maps are scaled according to the equations above.

Besides the set of VGV maps that are interpolated to simulate the effect of VGV angle on compressor performance, the lumped volume dynamic compressor model also calculates the flow area due to this angle setting, which affects the component dynamics. Thus, the flow area calculations due to VGV angle are also incorporated into the stage-by-stage model. The addition of the VGV model to the original volume dynamics model, ${ }^{2}$ which is also incorporated in this stage-by-stage model, is described in more detail in Ref. [9] for the compressor maps, and in Ref. [2] for the VGV effective area calculations.

\section{Stacking Compressor Stages}

The scaled down model ( $1 / 7^{\text {th }}$ for this VCE compressor) with appropriate boundary conditions will serve as the first upstream stage in developing the stage-by-stage model. The reason stages are connected in order, starting from upstream to downstream, is that this way the inlet conditions for each stage connected in this process will be known. Prior to stacking the stage-by-stage model, the Mach number estimator ${ }^{7}$ is disconnected and instead replaced with a constant Mach number calculated based on Eq. (11). This is because mismatches in the Mach number can cause instabilities in the simulation. The 
initial estimates for the cross sectional areas of the stages should be calculated as follows, by utilizing the corresponding stage Mach numbers obtained from Eq. (11).

$$
A_{n}=\frac{\dot{W}_{c, n}}{\rho_{s v, n} M_{n} \sqrt{\gamma R T_{S, n}}}
$$

The initial conditions for each subsequent stage connected are adjusted as described before, until the startup transients are minimized. For each step in this process, the final conditions from that run are also utilized to trim the corresponding area calculation for that stage, based on Eq. (12). When the startup transient is minimized, the Mach number estimator is reconnected for that stage, and the process is repeated for connecting the next downstream compressor stage and adjusting its cross section area and initial conditions, until all the stages have been successfully connected. When the Mach number estimator is connected for each stage, however, the Mach number should be verified and any final adjustments necessary for that stage should be made, before the next stage is connected.

Before starting the stacking process, the compressor stage is fine tuned to ensure that it produces the correct pressure and temperature at steady state at the desired operating point. The desired operating point would be the proper corrected speed for the operating condition utilized in the scaling process, at the proper operating point at that speed line-near the knee of the speed line at a certain stall margin/max efficiency point. The design speed for a particular stage is adjusted as follows, in order for the corrected speed to fall at the desired operating point, like the $100 \%$ corrected speed or whatever turns out to be a reasonable corrected speed for the cruise operating condition.

$$
N_{c, n}=\frac{N}{N_{d, n}} \sqrt{T_{r e f} / T_{t v, n-1}}
$$

The shaft speed is the same for all stages, but total temperature rises through each successive downstream stage. Hence, each stage will have a unique design speed in order to achieve a certain corrected speed ratio, according to Eq. (13). The reference temperature, $T_{\text {ref }}$, used is typically the sea level static temperature.

Figure 1 shows the pressure ratio operating point for stage 1 . Notice that the operating point is situated near the knee of the speed line, at a sufficient stability margin, but also at about the point where maximum operating efficiency would be found. For a generic map, if the operating point is placed further down, towards the high slope region of the speed line (high choking), the potential for instabilities increases. Such instabilities would not be due to stall or surge, but because the operating point moves into the highly choked region of the compressor, where, for relatively small changes in mass flowrate, there would be large changes in pressure ratio. For the desired pressure ratio of 1.419, in this case, the initial scaling put the operating point a little too low on the steep part of the speed line. Thus, the scale was adjusted slightly to put the operating point higher. A potential instability problem like the one that is described here for this $1 \mathrm{D}$ flow simulation would not be present in a $0 \mathrm{D}$ engine simulation. However, such instability behavior is expected to approximate the actual dynamics of a real compressor.

Efficiency maps have been scaled in this process based on Eq. (6) to try to ensure that for a desired pressure ratio the correct temperature ratio is produced. For adiabatic efficiency, the temperature ratio is related to pressure ratio and efficiency as

$$
T_{r}=1+\frac{P_{r, n}^{\left(\gamma_{c p}-1\right) / \gamma_{c p}}-1}{\eta_{c p}}
$$

The objective is for the stage-by-stage compressor model to produce the same overall temperature and pressure ratio as the lumped volume compressor, while operating at the same corrected speed, at about the same point on the speed line. However, it is found that scaling the maps as prescribed by Eq. (6), can cause the temperature ratio for the whole stage-by-stage compressor to deviate by a few percent from that of the corresponding lumped volume compressor model. A procedure that was found to work better is to calculate instead, the desired stage temperature ratio, $T_{r, s t}=\sqrt[J]{T_{r, l m p}}$. Then utilizing Eq. (14), with the desired stage temperature ratio, $T_{r, s t}$, and the desired stage pressure ratio, $P_{r, s t}$ from Eq. (5), calculate the desired stage efficiency, $\eta_{c p, s t}$ at this particular operating point. Next, by using the efficiency map of the lumped volume model, find the efficiency value, $\eta_{c p, l m p}$, at the operating point (for the speed line and the pressure ratio). Lastly, scale the lumped efficiency map by the ratio of $\eta_{c p, s t} / \eta_{c p, l m p}$ to produce the stage efficiency map that should provide for a close matching between the temperature ratios of the lumped volume and the stage-by-stage compressor models.

Some final trimming in the stage efficiency map may be required to more precisely match the temperature ratio, depending on the desired accuracy. Even though, for a dynamically accurate model, a few percent steady state error would not be significant. The reason that the objective here is to match the steady state performance of the lumped volume model is, as mentioned before, because the truth model here is considered to be the lumped volume model, whose development methodology 
has been verified against experimental data. ${ }^{7}$ If experimental compressor performance data are available, the likelihood is that these data are not stage-by-stage. Thus, in that case, the same procedure outlined here would be followed to develop a stageby-stage model of the experimental compressor, by first developing a lumped volume compressor model.

Corrected mass flowrate drives the performance maps, and as such it is important to know what corrected mass flowrate to expect from each stage, which can be calculated using the following equation.

$$
\dot{W}_{c m f, n}=\left(\dot{W}_{c, n}-\dot{W}_{b, n}\right) \frac{\sqrt{T_{t v, n-1} / T_{r e f}}}{P_{t v, n-1} / P_{r e f}}
$$

Because the inlet conditions for each stage will be different, each stage will have a unique scale factor for the corrected flowrate map, which will be the ratio of the corrected mass flowrate obtained in Eq. (15) to that of the lumped volume corrected mass flowrate.

\section{Stage-by-Stage Compressor Simulation Results}

Table 1 shows a steady state performance comparison between the lumped volume compressor model and the stage-by-stage model. Notice, all the errors are within a fraction of a percent. The objective is to develop a dynamically accurate model for closed loop propulsion system coupling to the AeroServoElastic (ASE) model of the vehicle to study APSE performance. The steady state accuracy of the model, as shown in Table 1, contributes to the dynamic accuracy in terms of low frequency offset error on the gain. For comparison, a $3 \mathrm{~dB}$ gain offset of the frequency response, which is not excessive, translates to $41 \%$ steady state error. Therefore, the errors displayed in this table are well within the typical accuracy sought for dynamic models, whose other important measures of accuracy are to maintain the frequency modes and damping of the system dynamics for gain and phase. The frequency response of the stage-by-stage model will be compared against the lumped volume model. However, the two are not expected to match, especially for the higher frequency range, and only a relative comparison will be performed here. Since no dynamic truth model response is available in this development for the stage-by-stage model.

\section{Parallel Flow Path Model}

\section{A. Stage Volume Dynamics}

The main purpose of developing a compressor parallel flow path model is to account for the effects of flow distortion entering the compressor on the compressor dynamics. In constructing this model, the compressor maps were also augmented to allow compressor operation in the stall-surge region, which can be done with either the parallel flow path model or with the stage-by-stage model, and will be described in the next section. The purpose of extending the compressor operation into the stall region is to model the onset of stall events, which would allow the model to closely capture the compressor dynamics when the compressor operates near stall conditions.

Starting from the stage-by-stage model (7 stages), the compressor stages are subdivided into quadrants as shown in Fig. 2. The paths do not necessarily need to be the same size, and the stages can be divided into more or fewer paths as desirable. As shown in Fig 2, the distortion pattern can be applied as different total conditions for each path at the inlet of the compressor. The last volume in Fig. 2 is a mixing volume, where the flow for the four paths comes together.

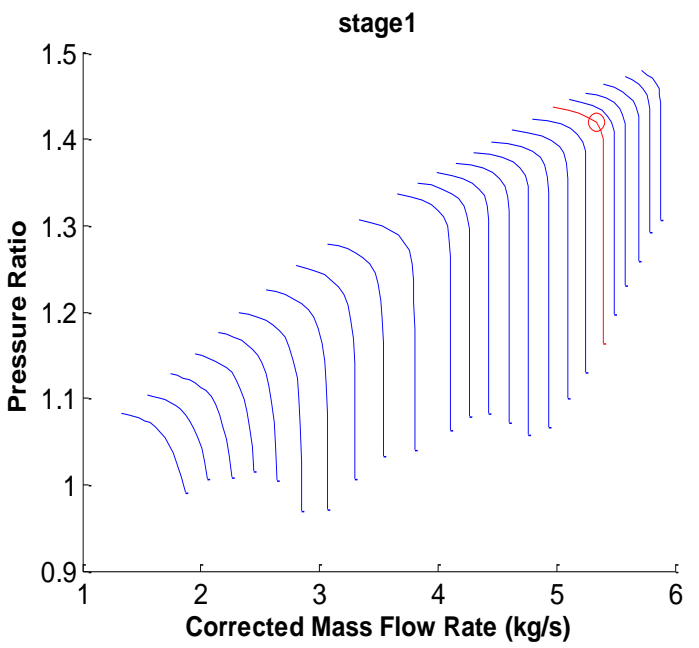

Figure 1. Pressure ratio map for stage 1. The circle indicates the operating point.

Table 1. Stage-by-stage and lumped volume compressor model steady state comparison

\begin{tabular}{|l|l|l|l|}
\hline Compressor & $\begin{array}{l}\text { Stage-by- } \\
\text { Stage }\end{array}$ & $\begin{array}{l}\text { Lump } \\
\text { Volume }\end{array}$ & $\begin{array}{l}\text { Percent } \\
\text { Error }\end{array}$ \\
\hline Pres. Out (Pa) & $1,219,493$ & $1,219,867$ & $0.031 \%$ \\
\hline Temp. Out (K) & 855.19 & 856.59 & $0.163 \%$ \\
\hline $\begin{array}{l}\text { Massrate In } \\
\text { (kg/s) }\end{array}$ & 18.43 & 18.43 & $0 \%$ \\
\hline
\end{tabular}

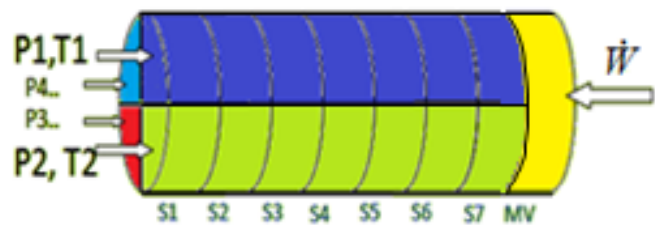

Figure 2. Pictorial diagram of a parallel flow 7-stage compressor model. $\mathrm{MV}$ is a mixing volume where the flows combine. 
As seen in Fig. 2, this model depicts 2-dimensional flow in cylindrical coordinates, axial (x-dimension) and rotational $(\varphi$ dimension). The boundary conditions set forth in this model, as shown in Fig. 2, are customary for subsonic flow in computational fluid dynamics (CFD). The stages could have also been subdivided in the radial dimension for a 3-dimensional flow model. This could have improved the accuracy of the model, especially for angle of attack (AOA) distortion effects. However, the added computational expense may not be justified in terms of added dynamic accuracy and there may be ways to compensate by averaging AOA flow distortion. Dynamic effects of the model due to radial dimension variation effects could, however, be a topic of future research.

Assuming again perfect gas, adiabatic and compressible flow, the Euler volume dynamics of the compressor stage as depicted in Eqs. (1) - (3) were recast in cylindrical coordinates in the axial and rotational dimensions, assuming that radial flow changes can be neglected.

$$
\begin{gathered}
\frac{\partial \rho_{s}}{\partial t}=-\frac{\partial\left(\rho_{s} u\right)}{\partial x}-\frac{1}{r} \frac{\partial\left(\rho_{s} w\right)}{\partial \varphi} \\
\frac{\partial\left(\rho_{s} u\right)}{\partial t}=-u \frac{\partial\left(\rho_{s} u\right)}{\partial x}-\frac{w}{r} \frac{\partial\left(\rho_{s} u\right)}{\partial \varphi}-\frac{\partial P_{s}}{\partial x} \\
\frac{\partial\left(\rho_{s} w\right)}{\partial t}=-u \frac{\partial\left(\rho_{s} w\right)}{\partial x}-\frac{w}{r} \frac{\partial\left(\rho_{s} w\right)}{\partial \varphi}-\frac{1}{r} \frac{\partial P_{s}}{\partial \varphi} \\
\frac{\partial}{\partial t}\left(\frac{P_{s}}{\gamma-1}+\frac{\rho V^{2}}{2}\right)=-\frac{\partial}{\partial x}\left[\left(\frac{\gamma P_{s} u}{\gamma-1}+\frac{\rho u^{3}}{2}\right)\right]-\frac{1}{r} \frac{\partial}{\partial \varphi}\left[\left(\frac{\gamma P_{s} w}{\gamma-1}+\frac{\rho w^{3}}{2}\right)\right]
\end{gathered}
$$

where Eq. (16) is the continuity equation, Eqs. (17) and (18) represent the axial and rotational momentum, and Eq. (19) is the energy equation. If Eqs. (16) - (19) are expressed in terms of states, $W$, fluxes, $F$, and source terms, $S$, the following equation is obtained.

$$
\frac{\partial}{\partial t}\left(W_{j}\right)=-a_{x j} \frac{\partial}{\partial x}\left(F_{x j}\right)-a_{\varphi j} \frac{\partial}{\partial \varphi}\left(F_{\varphi j}\right)+S_{j}
$$

where the state terms are the flow quantities in the left side of the Eqs. (16) - (19), the fluxes are represented by the derivative quantities in the parentheses of the first two terms on the right side of these equations, the source terms are the third terms, and the proportional $a$-terms are represented by the associated multiplicative expressions.

As necessary for engine component integration and associated interface boundary conditions, forward difference is maintained here to approximate spatial derivatives in the axial dimension, while due to the closed rotational domains, central difference is used to approximate spatial derivatives in the rotational dimension. Based on that, Eq. (20) can be expressed as follows.

$$
\frac{d}{d t}\left(W_{j, n, m}\right)=-a_{x j, n, m}\left(\frac{F_{x j, n+1, m}-F_{x j, n, m}}{\Delta x}\right)-a_{\varphi j, n, m}\left(\frac{F_{\varphi j, n, m+1}-F_{\varphi j, n, m-1}}{2 \Delta \varphi}\right)-\frac{S_{x j, n+1, m}-S_{x j, n, m}}{\Delta x}-\frac{S_{\varphi j, n, m+1}-S_{\varphi j, n, m-1}}{2 \Delta \varphi}
$$

It is assumed that by the time the flow gets into the combustor, after fuel is mixed, the flow should be fairly uniform with no remaining flow distortion effects. Any distortion downstream of the combustor would be instead due to pattern factor. The last volume in the model shown in Fig. 2, is a mixing volume. In general, a mixing volume at the component exit is appropriate when the component model is developed in isolation, and it can be removed later, if necessary, when parallel flow path component models are integrated together to form a system. Typically, in turbine engine design architectures, components are joined together with separating ducts. Even adjoining ducts can be modeled similarly as a parallel flow path in order to continue the modeling of downstream effects of flow distortion, starting from the inlet to the compressor exit or the combustor entry.

The mixing volume combines the outlet conditions of each path through the use of a weighted average and 1D volume dynamic equations, similar to those used in the stage-by-stage and lumped volume compressor models. The weighting factor, $\beta$, is proportional to the angular coverage of each parallel flow path. In this case, the four flow paths are selected to be equal in size, with the values of $\beta$ equal to $1 / 4$. The mixing volume mass flowrate going to each of the compressor paths is multiplied by this weighting factor to get $1 / 4^{\text {th }}$ the mixing volume mass flowrate to be distributed to each of the parallel flow paths. 
Maintaining generality, the weighting factor, $\beta$, could be selected to be different for each flow path, resulting in the following expressions for the continuity, momentum, and energy equations in the mixing volume:

$$
\begin{gathered}
\frac{d}{d t} \rho_{s, m v}=\frac{1}{V_{m v}}\left(\dot{W}_{m v}-\dot{W}_{c b}\right) \\
\frac{d}{d t} \dot{W}_{m v}=\frac{A_{m v} g}{l_{m v}}\left[\sum_{m=1}^{q}\left(\beta_{m} P_{t m, n=k}\right)-P_{t, m v}\right]\left(1+\frac{\gamma_{c p}-1}{2} M_{m v}^{2}\right)^{\frac{-\gamma_{c p}}{\gamma_{c p}-1}} \\
\frac{d}{d t}\left(\rho_{s, m v} T_{t, m v}\right)=\frac{\gamma_{m v}}{V_{m v}}\left[\dot{W}_{m v} \sum_{m=1}^{q}\left(\beta_{m}^{2} T_{t m, n=k}\right)-\dot{W}_{c b} T_{t, m v}\right]
\end{gathered}
$$

For the isolated compressor model, the mixing volume size is selected to be relatively small, comparable in size to or preferably smaller than an individual compressor stage. The reason is that the stage-by-stage dynamics for the parallel flow path compressor model should not be significantly affected by the inclusion of the mixing volume. Keep in mind that a smaller volume than that of the compressor stage will have an impact on the simulation maximum sampling time. The assumption here is that the flow is uniform by the time it reaches the compressor exit and as such the mass flowrates into the four flow paths of the $\mathrm{n}^{\text {th }}$ compressor stage are divided according to the weighting factor, $\beta$. Alternatively, the boundary conditions for the exit static pressures of all the flow paths could be assumed to be equal and Eqs. (22) - (24) could be modified accordingly. If no flow uniformity is assumed at the compressor exit, the exit pressures could be instead left free, and the mass flowrate of each path could be calculated according to the following relations: $\dot{W}_{m, n=k}=\beta_{m} \dot{W}_{m v} \frac{\Delta P_{m, n=k} A_{m, n=k}}{\sum_{m=1}^{q} \Delta P_{m, n=k} A_{m, n=k}}, \Delta P_{m, n=k}=$ $P_{m, n=k}-P_{m, n=k-1}$, where pressure in these expressions signifies static pressure.

\section{B. Stall Region Modeling}

For the parallel compressor model, the compressor maps as shown in Fig. 1 for the first stage, were extended into the stall region for each parallel sector in a compressor stage, in order to properly model compressor dynamics at or near stall operating conditions. This required modification of the stage characteristics calculations ${ }^{7}$ as well as extending the compressor maps into the stall region. Extended compressor maps can be seen in Fig. 3. The stall region was approximated by a straight line down to a minimum pressure ratio of one, at which point there is no mass flow. The maps as shown could have been further extended into the back flow region. However, an encompassing compressor simulation is not the purpose of this particular study. Efficiency in the stall region will trend towards zero percent as corrected mass flowrate drops. In the stall region, the drop in pressure is used as the driving force behind the simulation. Entering into the stall region will cause the pressure ratio, $P_{r}$, to be driven down to a minimum value of one in this case, in a delayed fashion, which can be approximated as
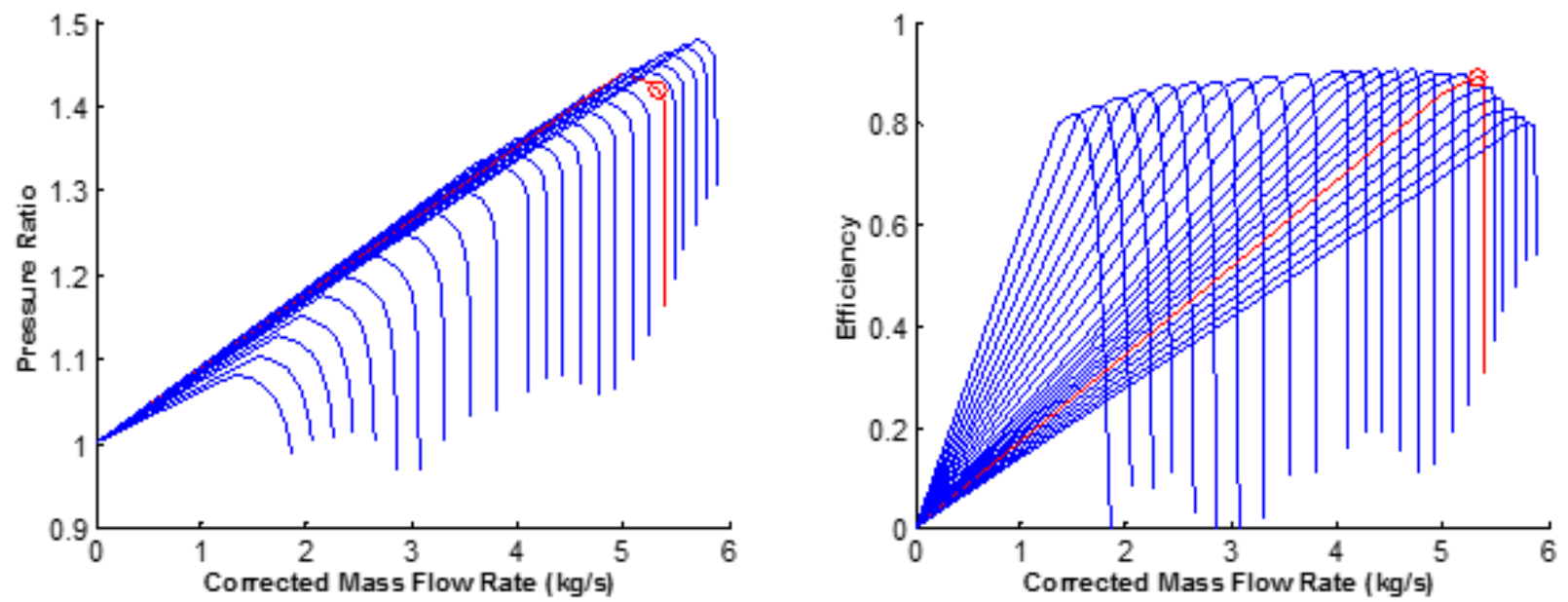

Figure 3. Example of compressor maps extended to the stall region-circle indicates the operating point at the particular corrected speed line; a) Pressure ratio map; b) efficiency map. 


$$
\frac{d}{d t} P_{r, \text { delay }}=\frac{1}{\tau}\left(P_{r}-P_{r, \text { delay }}\right)
$$

where $\tau$ signifies this time constant and $P_{r, \text { delay }}$ is the pressure ratio from this delayed process.

An appropriately sized nozzle, like a plug, was also added for both the stage-by-stage and parallel flow path compressor models in order to model the change in the mass flowrate due to changes in compressor outlet conditions. The nozzle was designed using the compressible flow equation based on the procedure outlined in Ref. [2].

\section{Results}

Figure 4 shows qualitative simulation comparisons between the stage-by-stage model developed in this paper and DYNTECC. ${ }^{5}$ This comparison is for the time it takes for compressor stages to move into stall conditions due to a pressure rise at the compressor inlet, which propagates downstream and to some degree amplifies, before the first downstream compressor stage stalls, followed by the rest. For the DYNTECC simulation, the figure shows the entrance static pressure to the stages, while for the simulation in this paper, it shows the actual static pressure of the stages. For the DYNTECC simulation, the input to stage 8 or stage 7 stalls first due to a pressure rise in stage 6 (not shown). For the DYNTECC simulation, which was developed from an experimental compressor, the simulation data produced the same pressure signatures that were observed experimentally. For the model developed in this paper, the entrance pressure to stage 5 or stage 4 stalls first (not shown). Even though the compressor geometries may not be the same (DYNTECC geometry is not known) and this compressor has seven stages, while DYNTECC's has ten, these results show that it takes approximately $17 \mathrm{msec}$ for the stages of each compressor to move into the stall condition, where pressure starts to drop. This overall time response, which is about the same for both compressors could signify that the stage geometries may not be appreciably different. The time constant for the pressure drop, when the compressor enters into stall conditions, was arbitrarily chosen for this simulation. Thus, this portion of the time response, which can easily be changed and adapted to an actual compressor stall response, is not meant to be compared for these two models. This comparison with DYNTECC, which has been verified against experimental data, validates to some degree this generic stageby-stage modeling approach.

For the parallel flow path compressor model, a pressure distortion of $500 \mathrm{~Pa}$ is applied to path 1 , at the inlet of the compressor, to see its response and how distortion propagates to the downstream compressor stages. Figure 5 show this response across multiple stages, with the distortion applied in both positive and negative directions. This level of distortion is not sufficient to move the compressor into the stall region. The negative part of the pulse has a more pronounced effect, especially for later stages, as the operating point moves further down from the knee and deeper into the steep or choked operating region of the compressor (Fig. 3). Operating in this region leads to instabilities for this dynamic simulation, which would also be expected to be the case for actual compressor operation, as small changes in corrected mass flowrate produce large fluctuations in the pressure ratio.

Proportionally, the later stages are progressively more affected by flow distortion as shown in Fig. 5, which is not what was originally expected. This result together with the results discussed for Fig. 4 negate the previous assumption that the flow could be assumed to be uniform by the time it reaches the compressor exit, and as such the exit pressure boundary conditions for the different paths could be assumed to be approximately the same. The later stages first enter into the stall operating region, which quickly causes the stall conditions to cascade to the upstream compressor stages. As seen in Fig. 5, a pressure distortion in path 1 primarily affects path 1 and 3 . Path 2 and 4 remain the same due to the fact that incoming flowrates originating from the mixing volume are all assumed to be the same, and as such the change in pressure and temperature have no effect on the mass flowrate. Based on
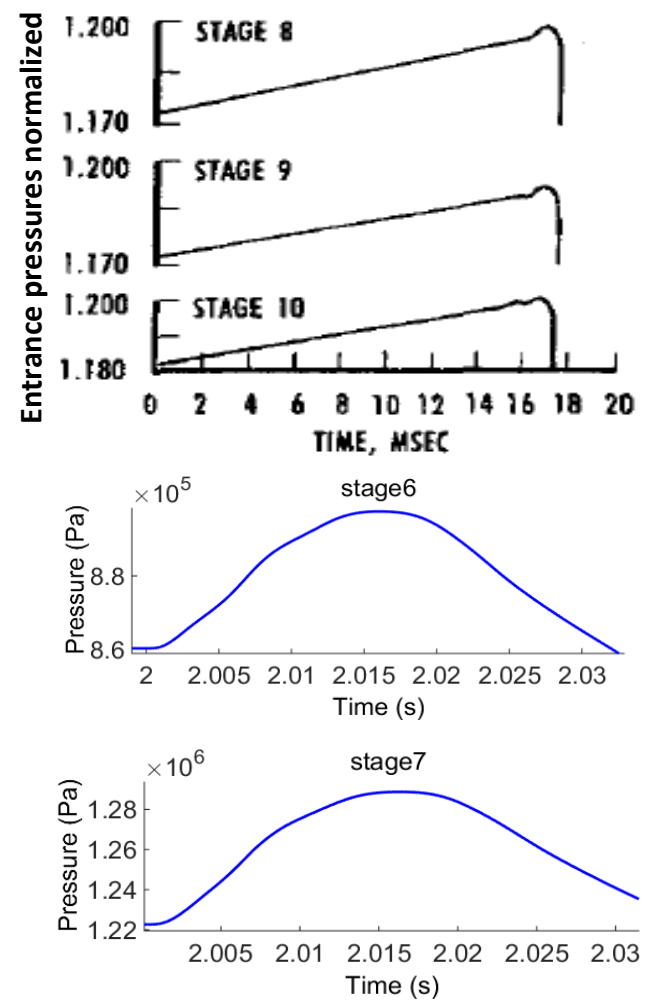

Figure 4. Relative comparisons for the time it takes for compressor stages to move into stall for DYNTECC (top 3 - copied from Ref. 5) and this compressor model (bottom 2) - the time constant for the pressure drop in stall is arbitrarily chosen in this case. 

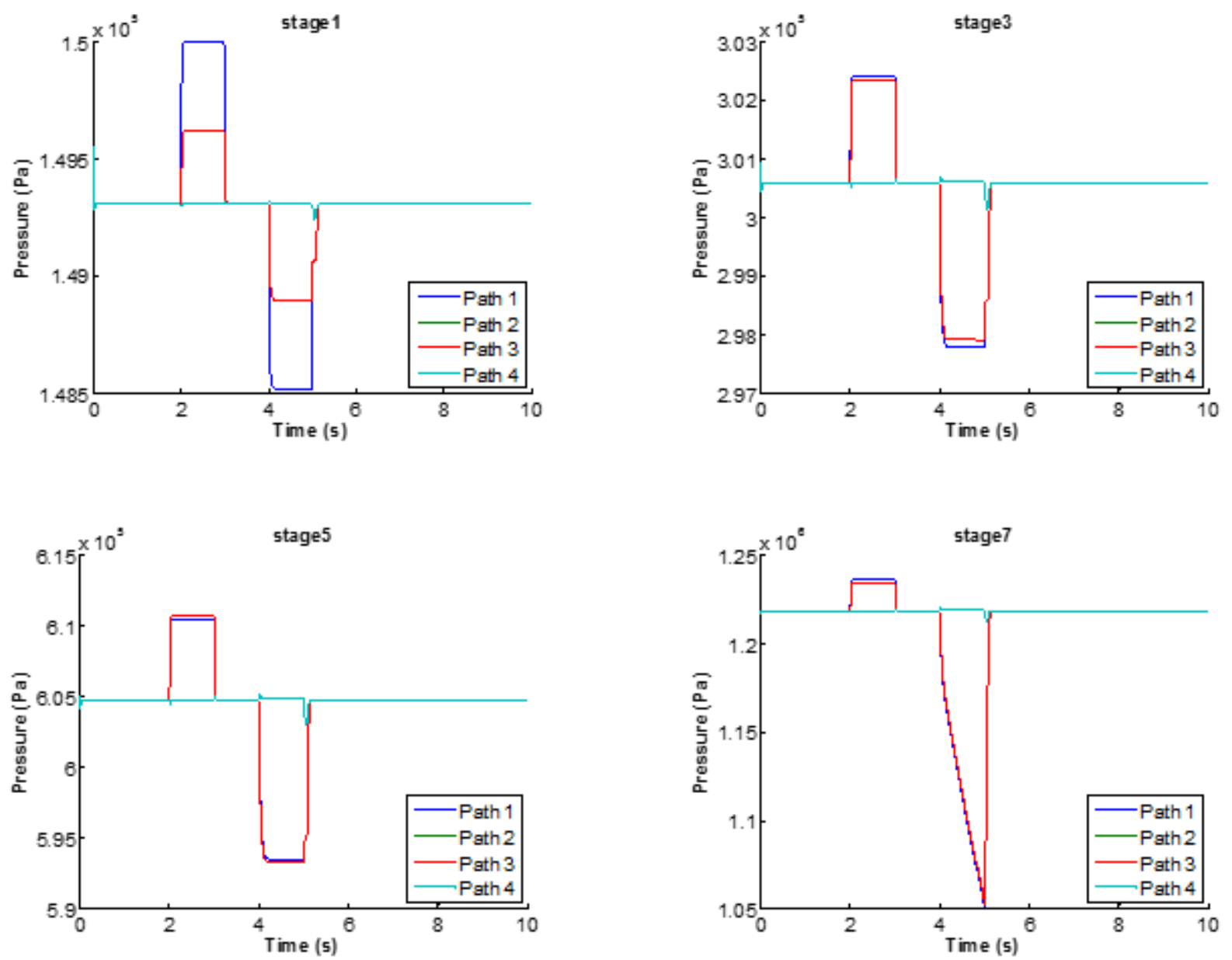

Figure 5. Pressure disturbance for various stages. Path 1 and 3 both experience a large drop in pressure ratio due to the pressure distortion pulse applied to path 1 at the inlet of the compressor.

how flow distortion affects different paths for this problem setup, and based on the fact that the flow is not well mixed by the time it gets to the compressor exit (in fact the opposite seems to be the case), the more appropriate boundary conditions at the compressor exit would be to let the mass flowrates vary as discussed in the previous section.

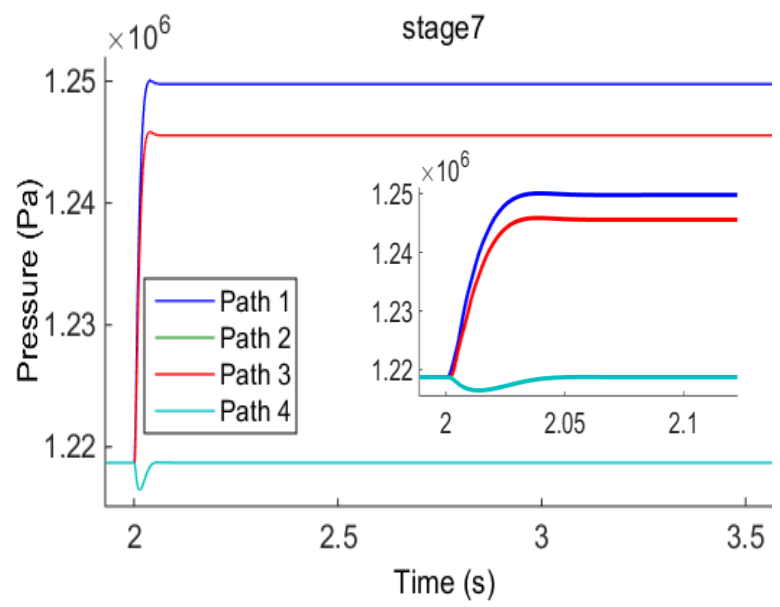

Figure 6. Pressure response at stage 7 to 1000 Pa step input into Path 1. Pressure increase in path 1 causes a similar increase in path 3 .

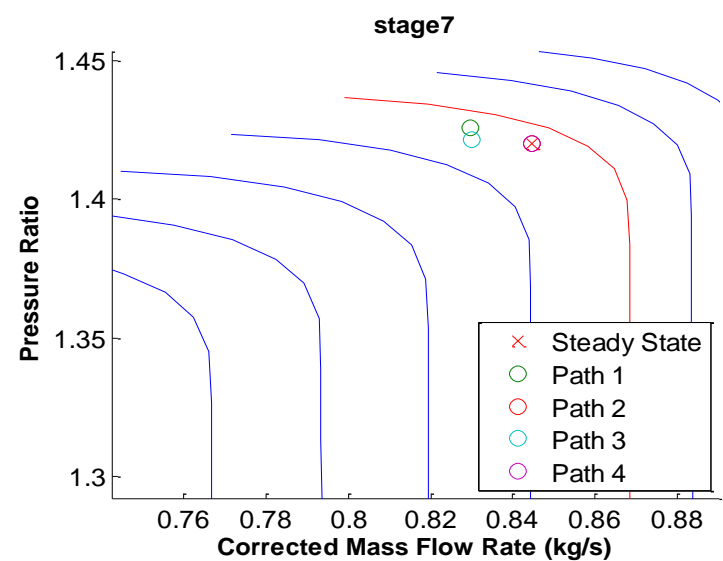

Figure 7. Operating point shift due to pressure step applied for Fig. 6. Operating points for path 1 and path 3 shift towards the stall line; path 2 and 4 remain the same. 
Figure 6 shows the pressure response to stage 7 for a $1000 \mathrm{~Pa}$ step applied at the compressor inlet to path 1 . The pressure increase in path 1 produces a similar increase in path 3 . The shift in the operating points can be seen in Fig. 7 . Both path 1 and path 3 move closer to the stall region, while path 2 and 4 remain the same. The time response as seen in the inset of Fig. 6 is relatively fast, less than $20 \mathrm{msec}$.

Figure 8 shows the pressure response to a $5000 \mathrm{~Pa}$ step applied at the compressor inlet to path 1, which is near the pressure distortion level that would cause the compressor to go into stall conditions. As seen in this case, the pressures for all four paths respond to this distortion level, where the pressures for path 1 and 3 move closer to stall (larger pressures) and the pressures for path 2 and 4 move away from stall.

Figure 9 show the response to a $5900 \mathrm{~Pa}$ step input into path 1 . It can be seen that this first pushes path 3 into the stall region, followed by the rest of the paths, as their pressure ratios are driven down. The stall of path 3 takes place shortly after distortion is applied at approximately $2 \mathrm{~s}$. Eventually, all the other paths stall as well, and surge occurs towards the end of the simulation time. This particular simulation becomes numerically unstable towards the end, due to rapid transients as surge takes place. If the exit mass flowrates of the individual paths were allowed to be different from each other, as discussed before, it could be expected that the results may look somewhat different.

Figure 10 shows the corrected mass flow rates of the four paths of stage 1. Comparing Fig. 9 with Fig. 10, it can be seen that for some of the paths, a downward trend in pressure is followed by a corresponding downward trend for their mass flow rates. This indicates that these paths have entered the stall region. On the other hand, a downward movement of the pressure in

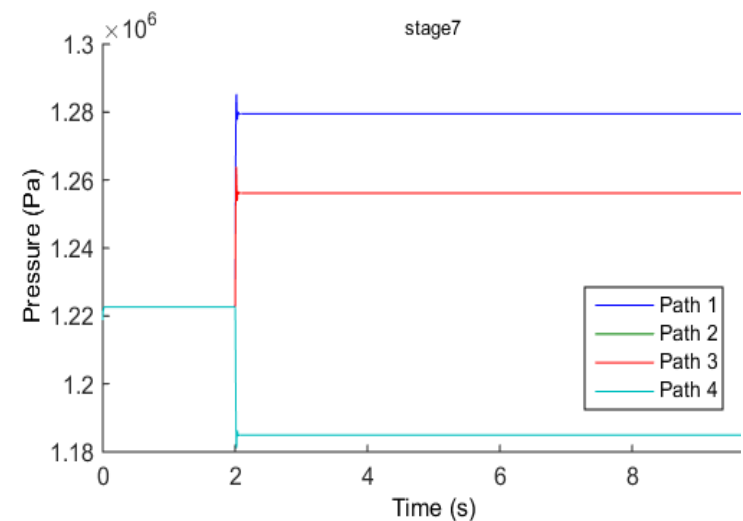

Figure 8. Pressure response to 5000 Pa step into path 1.

path 1 causes its corresponding mass flow rate to remain about constant. This indicates that unlike the other three paths, the initial trajectory of path 1 is in the choked region of the speed line. Eventually, however, all the paths experience surge oscillations. In this simulation, the surge time constant was arbitrarily chosen to be slow, compared to what would be normally the case, which allows the time responses of these trends to be seen a bit more clearly. Figure 11 shows a snapshot as the operating points of the flow paths of stage 7 transition into the stall region, which further clarifies comparisons between Fig. 9 and Fig. 10. In Fig. 11 path 2 operation is overshadowed by path 4 , as the snapshot is taken before the conditions for these two flows change as seen in Fig. 9 and Fig. 10.

In these simulations the rotating velocity of the flow is changing as well due to the distortion that is applied. A simulation of the rotational velocities in stage 1 of the compressor is shown in Fig. 12. This is for a relatively low amplitude distortion applied to path 1 , which does not cause stall. As seen in this figure, for a pressure pulse applied to path 1, the rotational velocities of path 2 and 4 are changing, compared to previous results that show that for the same pressure pulse the axial pressures of path 1 and 3 are primarily effected.

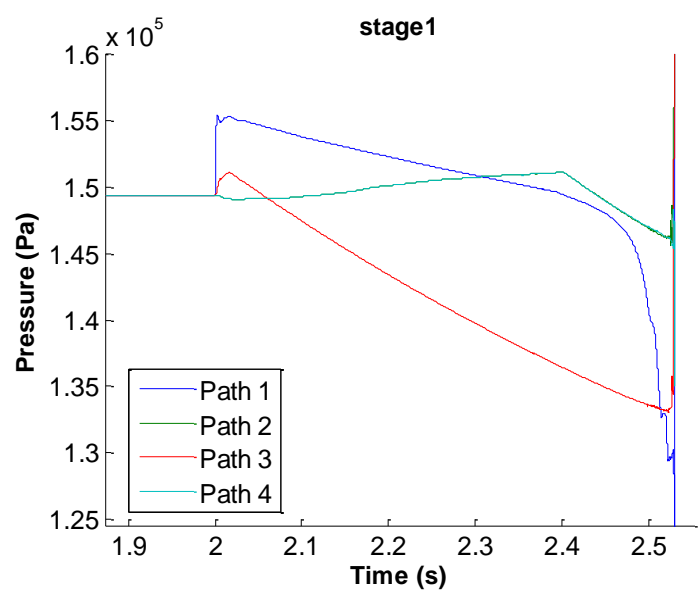

Figure 9. Pressure response during compressor stall. Path 3 enters stall region after a 5900 Pa step input into path 1.

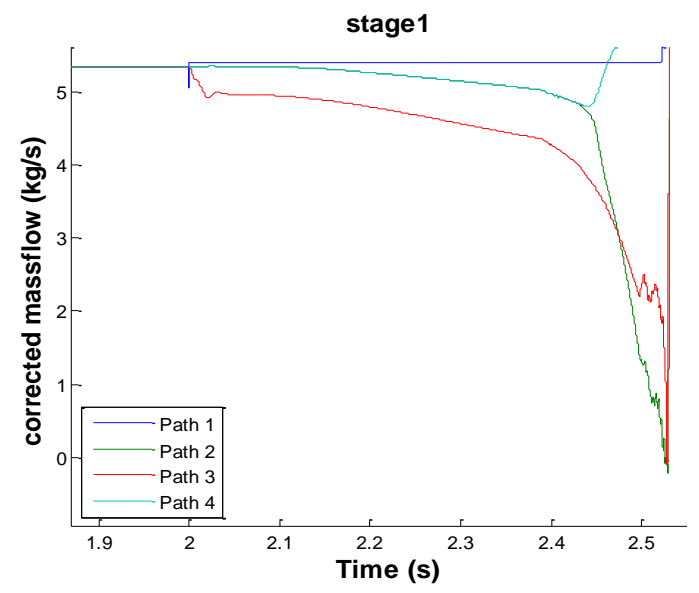

Figure 10. Mass flow rate response during compressor stall. Path 3 enters stall region after a 5900 Pa step input into path 1. 


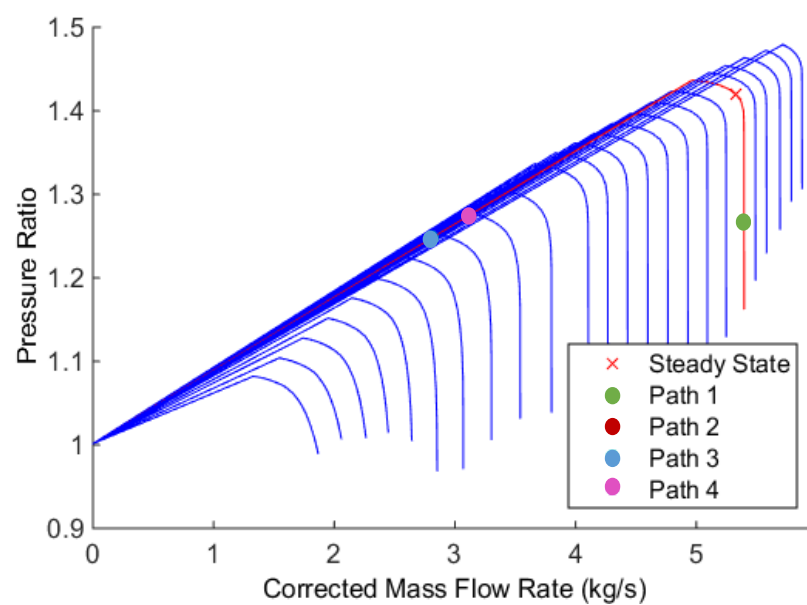

Figure 11. Snapshot of stage 7 operating points as the stage transitions into stall.

Applying distortion to a different path than path 1, produces correspondingly similar results. Also, applying temperature distortion produces similar results, albeit with different magnitudes.

Frequency responses for comparison were simulated for all three compressor models: the lumped volume, the stage-bystage, and the parallel flow path model. The frequency responses were generated by applying a relatively small pressure perturbation to the input of each simulation. The frequency was varied from about 1 to $3000 \mathrm{~Hz}$. These responses are shown in Fig. 13. As seen in this figure, there is a significant difference in the response between the lumped volume model and the other two, in both magnitude and phase. Compared to the lumped volume model, the other two models exhibit higher frequency dynamics. This is evident by comparing the flat frequency phase response at higher frequency for the lumped volume model to the corresponding downward trend for the other two models. This is expected, since compared to the lumped volume, the volumes of the multi-stage models are distributed smaller volumes, which would exhibit high frequency content. Up to approximately 60 $\mathrm{Hz}$ (the frequency range of interest for propulsion and the structural dynamics coupling for APSE), both the magnitude and the phase deviate significantly, by about $10 \mathrm{~dB}$ and $50^{\circ}$, respectively. This difference is more than sufficient to produce a stable vs. unstable overall APSE response. The difference in magnitudes between the stage-by-stage and the parallel flow compressor models is rather insignificant. The difference in phase, however, which is about $10^{\circ}$ at $60 \mathrm{~Hz}$, could be significant if the stability margin of the overall APSE response is relatively low, or if there are some time domain oscillations already existing at this frequency in an APSE simulation that utilizes a stage-by-stage compressor model. This frequency response comparison does not include the stall dynamics due to flow distortion, which could have a significant impact on propulsion and APSE. A parallel compressor model, however, that lets the mass flow rates be free at the outlet boundary as discussed before, should provide more concrete evidence to form these conclusions.

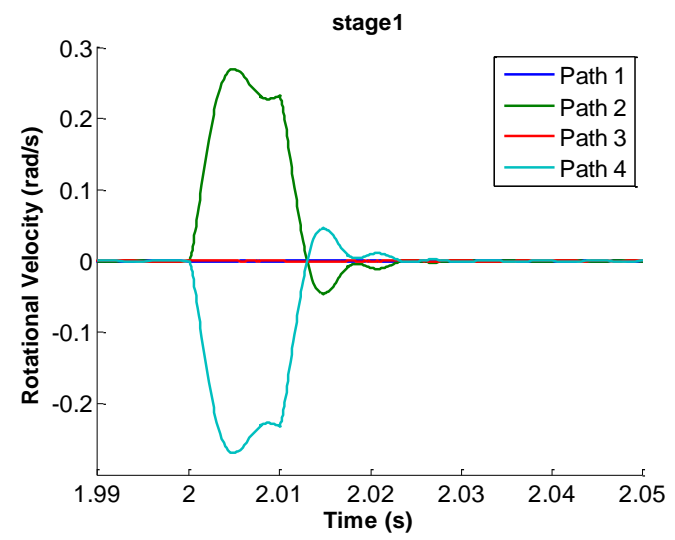

Figure 12. Stage 1 rotational velocities for a relatively small non-stalling pressure pulse applied to path 1.
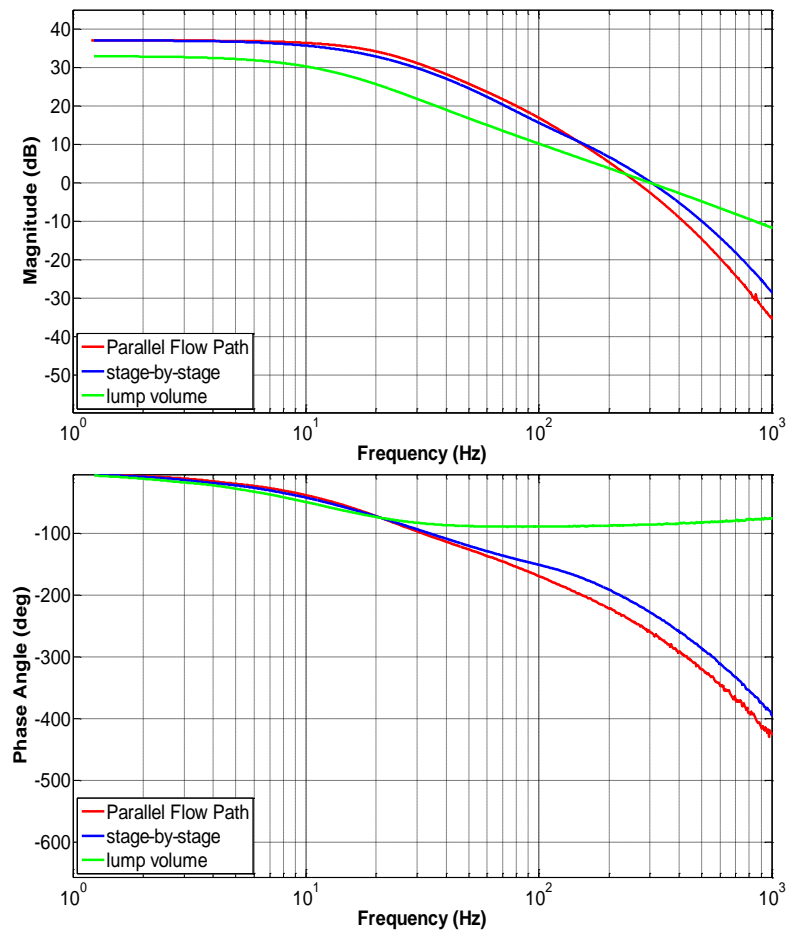

\section{Figure 13. Pressure frequency response} comparisons for the three compressor models. 


\section{Conclusions}

In this paper methodologies and formulations were introduced to help develop generic stage-by-stage and parallel flow dynamic compressor models. Even though the objective for developing these types of models is to apply them for analysis of AeroPropulsoServoElasticity performance for supersonic vehicles, the concepts introduced are rather generic. These methodologies have been systematically developed in an explicit way, which should be relatively easy to use and apply. The steady state results of the stage-by-stage and parallel flow path models are compared to the respective lumped volume dynamic model, whose development process has been verified previously against experimental results. The stage-by-stage model developed in this paper has been verified to a degree with a previous model developed and verified for an experimental compressor. For the parallel flow compressor model, there are no experimental data available to verify the model. Thus, at the moment, the trust in the accuracy of this model is placed on the soundness of the modeling methodologies developed and the results, which for the most part showed expected trends. However, more analysis would be needed to verify these models, especially, by modifying the compressor exit boundary conditions and repeating the analyses. This is based on the evidence that in response to distortion the flow does not seem to become uniform as the flow propagates into the downstream compressor stages, as was originally assumed. Preliminary results also indicate that the dynamics of the stage-by-stage and parallel flow path compressor models differ significantly from those of the lump volume compressor model, which may be evidence for the need of these higher fidelity propulsion component models to sufficiently assess AeroPropulsoServoElasticity performance.

\section{Acknowledgments}

The authors would like to express their gratitude to the Commercial Supersonic Technology (CST) Project under the NASA Advanced Air Vehicles Program (AAVP) for supporting this research effort.

\section{References}

1. Silva, W. A., et al. "The NASA High Speed ASE Project: Computational Analyses of a Low-Boom Supersonic Configuration," AIAA 2014-0675, AIAA SciTech Conference, Harbor Maryland, Jan. 13-17, 2014.

2. Kopasakis, G.; Connolly, J. W.; Seidel, J.; "Propulsion System Dynamic Modeling of the NASA Supersonic Concept Vehicle for AeroPropulsoServoElasticity," NASA-TN-16343, 2015.

3. Davis, M. W. Jr; O’Brien, W. F; "Stage-by-Stage Poststall Compression System Modeling Techniques," Journal of Propulsion and Power, Vol. 7, No. 6 (1991), pp. 997-1005.

4. Davis, M. W. Jr; "A Stage-by-Stage Poststall Compression System Modeling Technique: Methodology, Validation and Application,” Ph.D. Dissertation, Virginia Polytechnic Inst. and State Univ., Dec. 1986.

5. Hale, A. A; Davis, M. W. Jr; "Dynamic Turbine Engine Compressor Code, DYNTECC - Theory and Capabilities." AIAA 92-3190, AIAA Joint propulsion Conference, Nashville, TN, July 1992.

6. Greitzer, E. M.; "Surge and Rotating Stall in Axial Flow Compressor, Part I: Theoritical Compression System Model." ASME Transactions, Journal of Engineering for Power, Vol. 98, April 1976, pp 190-198.

7. Kopasakis, G.; Connolly, J. W.; Paxson, D. E.; Ma, P.; "Volume Dynamics Propulsion System Modeling for Supersonic Vehicle Research," GT2008-50524, ASME TurboExpo 2008, Berlin Germany; NASA/TM-215172; Journal of Turbomachinery, Vol. 132, October 2010.

8 Lytle, J. K.; "The Numerical Propulsion System Simulation: A Multidisciplinary Design System for Aerospace Vehicles," Tech. rep., NASA TM 1999-209194, 1999.

9. Connolly, J. W.; Friedlander, D.; Kopasakis, G; "Computational Fluid Dynamics Modeling of a Supersonic Nozzle and Integration into a Variable Cycle Engine Model," AIAA 2014-3687, AIAA Joint Propulsion Conference, Cleveland, Ohio, July 28-30, 2014. 